\title{
Care and Management of Burns Patients
}

\author{
Isa Sozen ${ }^{1 *}$, Dilsen Ornek ${ }^{2}$, Savas Baba ${ }^{1}$, Cem Emir Guldogan${ }^{1}$, Deniz Erdem², M Zafer Sabuncuoglu ${ }^{3}$, \\ A. Cinar Yasti ${ }^{1}$ \\ ${ }^{1}$ Ankara Numune Training and Research Hospital, Department of General Surgery, Burn and Intensive Care Unit Ankara, Turkey \\ ${ }^{2}$ Ankara Numune Training and Research Hospital, Department of Anesthesiology and Reanimation, Ankara, Turkey \\ ${ }^{3}$ Isparta Süleyman Demirel Üniversity, Department of General Surgery,Isparta, Turkey
}

*Corresponding author: Isa Sozen, Ankara Numune Training and Research Hospital, Department of General Surgery, Burns Treatment Center, Ankara Numune Training and Research Hospital, Ankara; Turkey, E-mail: isasozen2001@hotmail.com

Citation: Sozen, I., et al. Care and Management of Burns Patients. (2016) J Anesth Surg 3(1): 112- 113.

Received date: December 08, 2015, Accepted date: March 15, 2016, Published date: March 17, 2016

DOI: $10.15436 / 2377-1364.16 .030$

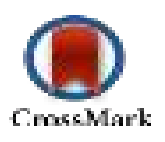

\section{Letter to Editor}

Burn injury is a significant health problem caused by the effect of heat, electricity and chemical substances, resulting in damages of different depths and extent. Determination of the degree of the burn depth is generally made clinically and with physical examination. In the acute phase, a burn, as a dynamic injury, may continue to deepen in the first seventy-two hours. In the determination of total body surface area burnt, rule of nines is widely used ${ }^{[1]}$. For burn patients, treatment should be planned specifically for burns after any emergency trauma intervention has been made. The success of shock treatment, early eschar excision and early grafting, appropriate and timely use of antibiotics to combat infection, new dressing materials and some new closure methods are positive developments providing good results in burn treatment. In addition to all these characteristics, vast majority of burns are suitable for treatment and follow-up in polyclinic conditions ${ }^{[1]}$. The care of serious burns patients can be challenging ${ }^{[1]}$. There is a need for long-term hospitalisation, a high degree of rehabilitation, psychiatry, physical therapy, consultations with a dietician and wound debridement which can cause severe pain. It must be anticipated that there could be pathophysiological changes in all the organs of these patients in the process from the initial burn to the completion of treatment. Mortality is not uncommon in patients admitted to the burns unit. High risk factors for death following a burn are age over 60 years, a burn of more than $40 \%$ of the body surface area and inhalation damage.

In inhalation damage and burns, extensive airway and lung oedema may rapidly develop. Morbidity and mortality increase in cases of inhalation damage. The mechanism of inhalation is complex and there may be a direct inhalation source of the face and upper airway or from the combination of hot gases. There may be chemical damage to the trachea, bronchi, alveoli and endothelium from the toxic products originating from a fire or injury to the oxygen transport with carbon monoxide and cyanide.

Electrical burns may cause greater morbidity than expected from the size of the burn area. Initial haemodynamic changes are a reduction in cardiac output and an increase in systemic vascular resistance. In addition, a hyperdynamic and hypermetabolic status may develop 2-5 days after large burns. Even if fluid resuscitation is made according to the guidelines, evaluation must be made according to the physiological status of the patient. In patients with serious burns with haemodynamic changes in the first 
12-36 hours, such as low cardiac output and hypervolemia, the response to fluid treatment is weak. Resuscitation monitorisation with urine output and vital signs is not appropriate in these patients ${ }^{[2,3]}$. Transpulmonary thermodilution monitorisation (TPTD) should be used with additonal biochemical parameters. Cardiac output, intrathoracic blood volume (ITBV), extravascular pulmonary fluid and other haemodyamic parameters can be evaluated with TPTD. Early resuscitation guidelines state that when the lactate level and haemodynamic targets are within safe normal limits, unnecessary fluid loading should be avoided. Excessive fluid can cause increased morbidity and mortality and a prolonged period of mechanical ventilation. It may also lead to compartment syndrome, an increase in the depth of the burn and pulmonary oedema. When the intrathoracic blood volume index is lower than $800 \mathrm{ml} / \mathrm{m}^{2}$, it is possible to reach a sufficient cardiac index $\left(2.2 \mathrm{~L} / \mathrm{min} / \mathrm{m}^{2}\right)$ and low lactate levels. Finally, fluid titration must be made according to the individual physiological response $\mathrm{e}^{[4,5]}$.

Large burns create extensive tissue destruction and with the activation of cytokine-dependent inflammatory mediators, damage is caused in areas both local to and distant from the burn. The systemic effects of the burn occur in 2 independent phases. The first is the burn shock phase and this is followed by the hypermetabolic phase. When the burn area exceeds $25 \%$ $30 \%$ of the total body surface, widespread oedema is seen in undamaged tissues. Plasma loss from burned tissue develops in the first 48 hours or later. Intravascular fluid loss from the burn area and oedema in areas not burned, result in burn shock causing insufficient tissue and organ perfusion. Burn-induced fluid loss is seen more with the loss of erythrocyte volume than haemoconcentration anaemia ${ }^{[3]}$.

There are changes in the pharmacodynamic and pharmacokinetic responses to medication in large burns. Painkillers and sedatives are required at higher doses than normal by burns patients. Opioids are an inevitable medication for these patients and the need of these patients for opioids is increased. However, as systemic absorption is reduced, tissue perfusion reduces and intramuscular or transdermal use of opioids may not be appropriate in burns patients. Patient-controlled analgesia gives extremely good results. Methadone has been found to be effective in neuropathic pain which would prevent withdrawal of seratonin and neurepinephrine. Very short-term effect opioids such as alfentanil and remifentanil are recommended during wound debridement. There are studies showing that clonidine is effective. NSAIDs can be used paying attention to renal functions. Acetaminophen is a good alternative. Ketamine has an important role in the anaesthesia method for these patients and it may be used orally and parenterally in patients who do not respond to opioid treatment. The use of major tranquilisers such as haloperidol and sedatives such as benzodiazepines may be useful in addition to analgesics in patients with high levels of fear and anxiety. Antihistamines are recommended for anxiety, pain and itching. Polymodal C fibres in contact with itching wounds create problems in the recovery period. Epidural techniques, nerve blocks and subcutaneous infiltration techniques are also used in pain management. In those with very severe pain, general anaesthesia can be applied ${ }^{[1-5]}$.

Burns are classified according to the depth of the injury. In superficial second-degree burns, the epidermis and the superficial dermis are mainly affected. These kinds of burns are very painful. Chronic pain may develop in the vast majority of patients. Acute nociceptive pain and peripheral nerve damage causes wind-up and central sensitisation and may cause secondary hyperalgesia. This can be corrected by aggressive pain treatment. Even if local anaesthetics seem to be effective in the treatment of burns pain, it is recommended that they are applied with care paid to the systemic toxicity from the epidermis with corrupted integrity ${ }^{[1-6]}$.

Because of burns are one of the most widespread injuries in accidents and remain a global public health issue the need for a local and effective wound healing medication efficent in preventing infection, decreasing fluid imbalance, promoting reepithelialization, reducing the occurrence of scar tissue and is still an important challenge ${ }^{[6]}$.

This short paper, in the manner of the iceberg model, only includes the upper points of the difficulties faced in the care of burns patients. The use of current guidelines together with clinical experience and observation can be considered to make a contribution to the reduction of morbidity and mortality in these patients.

\section{References}

1. Sozen, I., Guldogan, C.E., Kismet, K., et al. Outpatient burn management and unnecessary referrals. (2015) Ulus Travma Acil Cerrahi Derg 21(1): 27-33.

2. Lundy, J.B., Chung, K.K., Pamplin, J.C., et al. Update on Severe Burn Management for the Intensivist. (2015) J Intensive Care Med.

3. Bittner, E.A., Shank, E., Woodson, L., et al. Acute and Perioperative Care of the Burn-Injured Patient. (2015) Anesthesiology 122(2): 448-464.

4. Arlati, S., Storti, E., Pradella, V., et al. Decreased fluid volume to reduce organ damage: a new approach to burn shock resuscitation? A preliminary study. (2007) Resuscitation 72(3): 371-378. 5. Sanchez, M., Lorenzo, A.G., Herrero, E., et al. A protocol for resuscitation of severe burn patients guided by transpulmonary thermodilution and lactate levels: a 3-year prospective cohort study. (2013) Crit Care 17(4): R176.

6. Halil, K., Gokdemir, M.T., Sogut, O. et al. Effects of folk medicinal plant extract ankaferd blood stopper on burn wound healing. (2013) Acta Medica Mediterranea 29: 497. 